\title{
IDENTIFICACIÓN ODONTOLÓGICA FORENSE: REVISIÓN DE LA LITERATURA Y REPORTE DE UN CASO *
}

\author{
${ }^{1}$ Freddy Moreno G., ${ }^{2}$ Sandra Moreno C., ${ }^{3}$ Liliana Marín J. \\ ${ }^{1}$ Odontólogo, Docente, $U$. del Valle. ${ }^{2}$ Odontóloga, Docente, U. del Valle. ${ }^{3}$ Odontóloga, \\ Especialista en Criminalística y Ciencias Forenses.
}

Autor responsable de correspondencia: Freddy Moreno Gómez

Dirección electrónica: freddymg@univalle.edu.co

\section{RESUMEN}

Este artículo resalta el papel de la odontología dentro del campo de las ciencias forenses en los procesos de identificación de cadáveres y restos humanos en avanzado estado de descomposición o esqueletizados. Así mismo, se exalta la importancia de la Historia Clínica Odontológica y su respectiva Carta Dental, las cuales deben ser diligenciadas durante cualquier consulta o atención odontológica con el fin de proporcionar la mayor cantidad de características e información antemortem de un individuo, en caso que las autoridades competentes lo requieran. [Moreno F, Moreno S, Marín L. Identificación odontológica forense: Revisión de la literatura y reporte de un caso. Revista Ustasalud Odontología 2007; 6: 60 - 66]

Palabras clave: Odontología forense, Identificación dental, Historia Clínica Odontológica, Carta Dental. Cotejo dental ante postmortem.

\section{FORENSIC DENTISTRY IDENTIFICATION: LITERATURE REVIEW AND A CASE REPORT}

\begin{abstract}
This literature review and report of a case emphasizes the role of dentistry in forensic sciences and in the process of corpse identification in advanced state of decomposition status. Also it raises the importance of the dental clinical record and the dental chart, which must be filled before making any clinical attention of patients in order to provide the greater amount of information and characteristics of an individual antemortem in case those legal authorities require it.
\end{abstract}

Key words: Forensic dentistry, Dental identification, Dental clinical record, Dental chart, Ante - postmortem comparison.

Recibido para publicación: 26 de abril de 2007. Aceptado para publicación: 4 de julio de 2007.

* Grupo de Investigación Cirugía Oral y Maxilofacial de la Universidad del Valle

\section{INTRODUCCIÓN}

La odontología, basada en el trabajo integral técnico y científico junto con otras disciplinas (medicina, patología, antropología, dactiloscopia, entomología, biología, química, física, balística y derecho entre otras) dentro del contexto de las ciencias forenses, realiza notables aportes en procura de una recta administración de la justicia con principios éticos y morales. ${ }^{1}$

Pederson define la odontología forense como la rama de la odontología que trata del manejo y el examen adecuado de la evidencia dental y de la valoración y la presentación apropiada de los hallazgos dentales en interés de la justicia. ${ }^{2}$ Aprile la ubica como una rama de la medicina legal que trata sobre la aplicación de los conocimientos odontológicos al servicio de la justicia. ${ }^{3}$ Montiel-Sosa la describe como el estudio de las características de las piezas y arreglos dentales para hacer comparaciones de historias clínicas testigos y establecer la identidad de las personas y restos humanos. ${ }^{4}$ En un sentido más amplio, Guerra define a la odontoestomatología forense como el peritaje forense que hace el odontólogo al tomar como base las evidencias que puede ofrecer el sistema estomatognático, dado que la aplicación de los conocimientos odontológicos puede contribuir a la resolución de problemas jurídicos. ${ }^{3,5}$ 
En Colombia, el estado del arte de la odontología forense avanza en virtud de la convulsionada situación social y política que ha acontecido en el país durante los últimos 30 años, ${ }^{3}$ en donde las muertes violentas, las masacres, los ataques terroristas, la práctica generalizada de la desaparición forzada, la inhumación de los cuerpos sin vida en fosas comunes, la cremación postmortem de los cadáveres, la eliminación de las huellas dactilares y la destrucción de los dientes se convierten en obstáculos que dificultan el proceso de identificación; ${ }^{6}$ razones por las cuales el odontólogo dentro de su labor como auxiliar de la justicia, en su actuar como perito de acuerdo a las leyes colombianas $\mathrm{s}^{7-9} \mathrm{y}$ consecuente con el nuevo sistema acusatorio el cual debe entrar en vigencia en todo el territorio nacional para el 31 de diciembre del 2008 , debe aportar sus conocimientos a las ciencias forenses, principalmente en el campo de las lesiones personales, el dictamen de responsabilidad profesional, los grandes desastres, la identificación dental ante y postmortem, la identificación y clasificación de las huellas de mordida por las arcadas dentales humanas, el abuso y maltrato infantil y el estudio de restos humanos para el dictamen de la edad y el sexo, ,3,50-16 cuando entidades oficiales (Fiscalía, Procuraduría, Defensoría, Consejería Presidencial, jueces de la República), organizaciones no gubernamentales $\left(O N G^{\prime} s\right)$ y entidades internacionales (INTERPOL, Amnisty Internacional, Comitte of Freedom and Responsability, ONU) requieran de sus conocimientos.

Este desempeño se hace evidente ya que es a través de los dientes que se puede obtener información sobre: (1) la edad, mediante el estudio de los patrones de erupción de los dientes y el desgaste dental; ${ }^{17,18}$ (2) el sexo, tras la obtención de ADN genómico de la pulpa dental para extraer amelogenina, un gen ligado al género que determina el perfil XX o XY mediante la prueba de reacción en cadena de la polimerasa PCR; ;9,20 (3) la influencia de grupos poblacionales en la expresión y variabilidad de rasgos

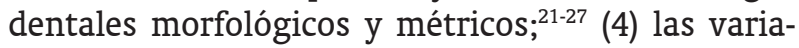
ciones individuales de los dientes reportadas como anomalías de forma, tamaño, número y posición; ${ }^{20,28}$ (5) los hábitos alimenticios, mediante el estudio y comprensión de la dieta, fitolitos, elementos traza, isótopos estables y tinciones extrínsecas; ${ }^{28-30}$ (6) los procesos culturales resultantes del empleo de los dientes como herramienta, estrés ocupacional, piercing, labretts, expansores, joyas dentales y tallado con fines bélicos, religiosos y estéticos; ${ }^{17,28,31-34}$ (7) los procesos patológicos como alteraciones en el desarrollo del esmalte y/o dentina, caries, bruxismo, enfermedad periodontal y enfermedad pulpar; ${ }^{10,35}(8)$ los traumas dentales tipo fracturas coronales y radi- culares, abrasiones, atriciones y abfracciones; ${ }^{10,36} \mathrm{y}$ las alteraciones de los dientes ocasionadas durante la práctica odontológica tales como extracciones, endodoncias, rehabilitaciones, ortodoncia, restauraciones, implantes e inclusive la iatrogenia que se pueda causar durante algún tratamiento terapéutico. ${ }^{36}$ Además, todo el sistema estomatognático puede brindar información al ser analizadas las estructuras anatómicas óseas de la maxila y la mandíbula, alvéolos, crestas óseas, planos antropométricos, radiografías intra y extraorales, rugas palatinas, forma de los arcos dentales, forma y surcos de los labios, patrón de mordida, torus palatinos y linguales por mencionar algunos. . $^{3-5,18,37-40}$

Todo esto se encuentra fortalecido científica y técnicamente debido a las propiedades tafonómicas (resistencia al medio ambiente) de los dientes, y si se tiene en cuenta que pese a las condiciones extremas de muerte, los dientes son las estructuras que mejor se conservan dado que el esmalte es el tejido mas resistente del cuerpo humano, ${ }^{41,42}$ preservándose en situaciones extremas como $\mathrm{pH}$, salinidad, humedad, altas temperaturas. ${ }^{3,43-45}$ De igual forma, la anatomía de los dientes es expresada genéticamente siendo única e irrepetible en cada diente y en cada individuo, ${ }^{46}$ y la estructura dental (métrica y morfológica) histo-embriológicamente constituida no cambia por acción propia ni estará sujeta a procesos de remodelación como sucede con el hueso, excluye el desgaste mecánico o la atrición de las superficies oclusales y acumulación de dentina secundaria. ${ }^{47}$

Por tanto, el reporte de este caso, evidencia la importancia del ejercicio de la odontología forense durante el proceso de identificación de un individuo mediante el cotejo del examen postmortem con la Historia Clínica Odontológica postmortem, durante el cual se empleó el estudio de las características fisio-patológicas del sistema estomatognático, el análisis de los dientes y los tratamientos odontológicos realizados, principalmente una prótesis parcial acrílica mucosoportada.

\section{Identificación odontológica}

Los procesos de identificación son una de las principales actividades que realiza la odontología dentro de las ciencias forenses. ${ }^{48}$ De acuerdo al Diccionario de la Real Academia de la Lengua Española identificar es "reconocer que una persona o cosa es la misma que se supone o se busca". Rodríguez indica que la identificación es un proceso comparativo y reconstructivo tendiente a ubicar a una persona desconocida dentro de un universo bio-social conocido. ${ }^{47}$ Citados por Gil y colaboradores, ${ }^{41}$ Locard define la identificación personal como la operación policial o medico-legal mediante la cual se establece la 
personalidad de un individuo, en donde la identidad se constituye en el conjunto de caracteres por los cuales el individuo define su personalidad propia y se distingue de sus semejantes; y Lacassagne entiende que es la determinación del conjunto de signos que distinguen a un individuo de todos los demás, ya sea durante la vida o después de la muerte. Mientras que, de manera más específica, Carrera-Carbajo se refiere a la necroidentificación como la comparación, cotejo o correlación de los datos obtenidos del cadáver (postmortem) con aquellos obtenidos en la investigación criminal (antemortem) en donde se busca la coincidencia de ambos datos para obtener la identidad de un individuo. Mertz se refiere respecto a la identificación odontológica como la comparación de registros antemortem con los registros postmortem proporcionando al odontólogo forense características distintivas suficientes para identificar a una persona. ${ }^{28}$

Con base en estas definiciones, la legislación Colombiana ha unificado la dactiloscopia y ha adoptado la Carta Dental como sistemas de identificación, ${ }^{7}$ y aunque carece de reglamentación, indica que la elaboración de la Historia Clínica es obligatoria en todos los consultorios odontológicos, privados y públicos, y que podrá ser proporcionada, tal como se manifestó, a las autoridades competentes cuando éstas lo requieran. Bajo este soporte legal, la odontología puede contribuir al desarrollo de la justicia dentro de los procesos de identificación dental ante y postmortem de individuos y de restos humanos, a través de pruebas periciales o documentales con el fin de demostrar que una persona o cadáver es quien verdaderamente es o que unos restos humanos son y/o pertenecen a una persona y no a otra. ${ }^{3}$

Por ello, se puede comprender que cada diente es un órgano que representa a un individuo y tal como Rodríguez indicó, "los dientes hablan y cuentan la historia de la persona desaparecida que en vida formó parte de un medio ambiente, un grupo social determinado y con una individualidad que lo hace único e irrepetible". ${ }^{17}$

En términos generales, la identificación odontológica se basa en la comparación de registros antemortem con los registros postmortem que proporcionan al odontólogo forense características distintivas suficientes para identificar a una persona. Tales características se sustentan científicamente en la individualidad morfológica del esqueleto y de los dientes que permiten distinguir a una persona por sus rasgos propios. ${ }^{4,28}$ Este proceso de identificación puede ser compara- tivo lo cual permite cotejar los registros odontológicos antemortem (historia clínica, odontograma, periodontograma, radiografías, modelos de estudio, análisis cefalométricos, plan de tratamiento y otros), postmortem (información que se obtiene del cadáver) y reconstructivo (cadáveres en avanzado estado de descomposición, carbonizados o en reducción esquelética o restos humanos que requieren una reconstrucción facial. ${ }^{1,3,12}$ Sin embargo, el cotejo que realice el odontólogo forense a partir de la información proporcionada de la Historia Clínica puede generar resultados fehacientes a partir de la aplicación de métodos científicos legales como resultado de la comparación de evidencias dubitadas e indubitadas; e indiciaría o complementaría, la cual recopila y compara los datos biográficos, antecedentes médicos, prendas de vestir, algunos documentos y señales particulares con métodos no científicos.

A partir de estos resultados, la American Board of Forensic Odontology clasifica el proceso de identificación a partir de las estructuras que conforman el sistema estomatognático de acuerdo a cuatro posibilidades: (1) identificación positiva cuando la información antemortem es suficiente y claramente comparable con los datos obtenidos postmortem; (2) identificación posible cuando la información antemortem obtenida no es suficiente para establecer comparaciones precisas; (3) evidencia insuficiente cuando la información obtenida ante y postmortem no representa un soporte fehaciente para la identificación; y (4) exclusión, resultado a la incompatibilidad de la información ante y postmortem. ${ }^{49}$

\section{REPORTE DE UN CASO}

Fue reportado como desaparecido ante las autoridades competentes, un individuo víctima del conflicto armado que acontece actualmente en Colombia. En respuesta se desencadena un mecanismo de búsqueda en las bases de datos de desaparecidos con resultados negativos. Posteriormente, a través de información suministrada por los habitantes del lugar, se encuentra en una vereda, un cadáver inhumado en avanzado estado descomposición de género masculino y que por sus características de contextura fisica, pertenencias y prendas de vestir guiaba a una identificación indiciaria del individuo reportado como desaparecido (Figuras 1 y 2). Una vez en la morgue, se realizó por parte del odontólogo forense la autopsia oral, que permitió obtener información postmortem de características individualizantes en tejidos óseos y dentales, al igual que tratamientos odontológicos tales como una prótesis parcial acrílica mucosoportada con un refuerzo de rejilla metálica en el paladar además de una incrus- 
tación metálica en el ángulo disto-vestíbulo-palatino del diente artificial que reemplaza al incisivo lateral superior derecho (Figuras 3 y 4), aspecto protésico que resulta inusual y que se relaciona con alteraciones estéticas socio-culturales. De igual forma se describió la pérdida antemortem de molares inferiores, la pérdida postmortem del incisivos central superior derecho, pérdidas óseas horizontales y verticales generalizadas compatibles con enfermedad periodontal y resto radicular del primer premolar superior derecho cuya cortical ósea vestibular presentaba una fenestración compatible con una fístula de drenaje por absceso periapical (Figura 5).

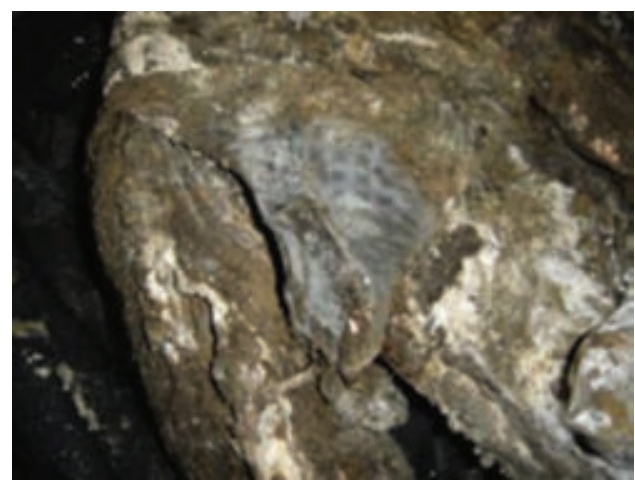

Figura 1. Restos humanos en avanzado estado de descomposición en los cuales se aprecian restos de las prendas de vestir.

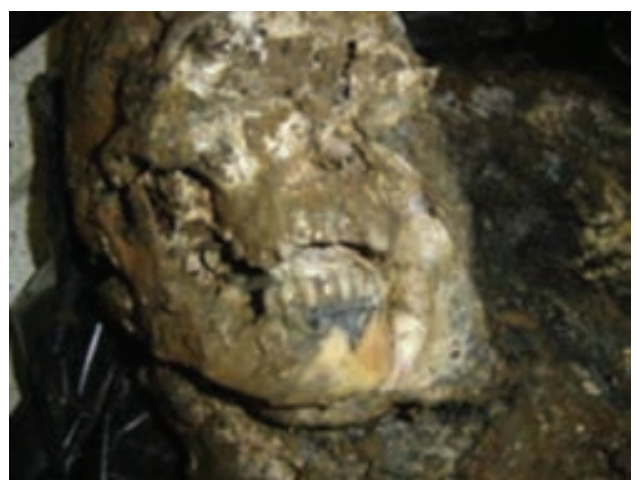

Figura 2. Aspecto de los tejidos periorales en avanzado estado de descomposición.

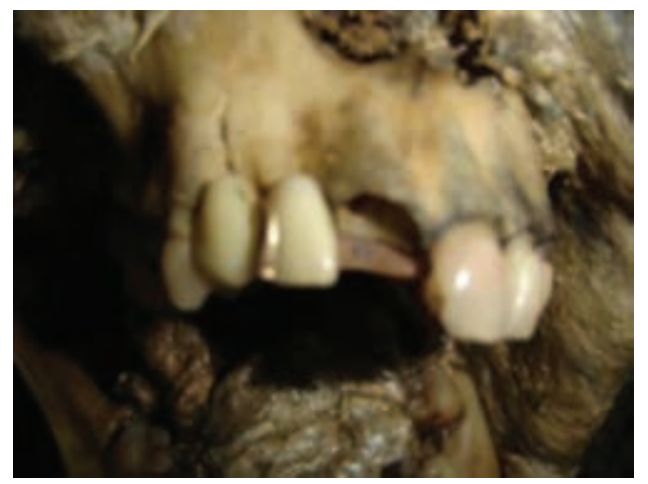

Figura 3. Prótesis parcial acrílica mucosoportada in situ, en la cual se observa una incrustación metálica en distal del incisivo lateral superior derecho. Hay pérdida postmortem del incisivo central superior derecho.

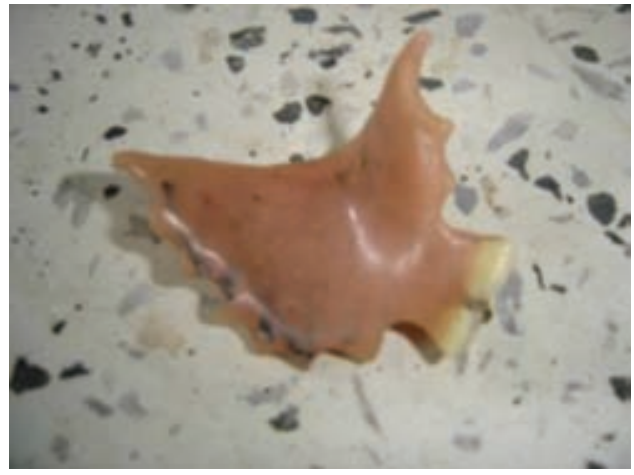

Figura 4. Prótesis parcial acrílica mucosoportada.Se puede observar la rejilla metálica de refuerzo y dos dientes artificiales que reemplazan el incisivo lateral superior derecho y el canino superior derecho.

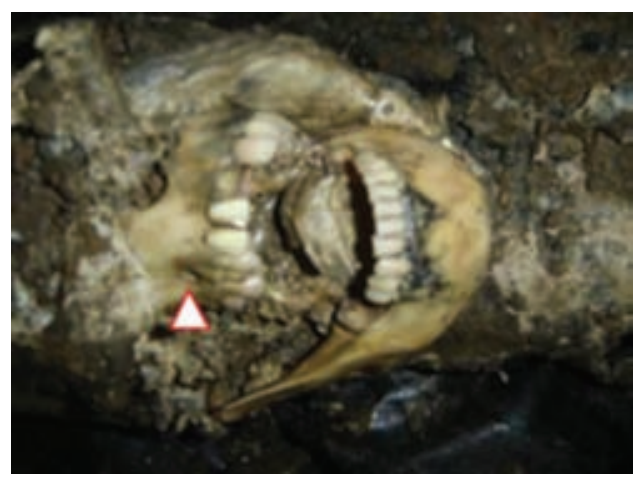

Figura 5. Fenestración ósea compatible con fístula de drenaje por absceso periapical de resto radicular del primer premolar superior derecho, pérdidas dentales antemortem y pérdidas óseas verticales y horizontales compatibles con enfermedad periodontal.

El cotejo odontológico se realizó teniendo en cuenta la historia clínica antemortem, la cual fue obtenida con base en la información suministrada por la familia. No se contó con documentos anexos (radiografías, modelos de estudio, órdenes de laboratorio). Para este caso se tuvo en cuenta la posición de los dientes y los tratamientos odontológicos realizados (prótesis parcial acrílica mucosoportada, rejilla de refuerzo en la prótesis, incrustación metálica en el póntico que reemplaza al incisivo lateral superior derecho, ausencia del incisivo central superior derecho), lo cual brindó suficientes concordancias absolutas para lograr la identificación positiva de la persona.

\section{DISCUSIÓN}

Tal como lo describe el Instituto Nacional de Medicina Legal y Ciencias, ${ }^{15}$ la identificación científicocriminalística de la muerte requiere una metodología de trabajo integrado que incluye la compilación, análisis, interpretación e intercambio de información por parte de los investigadores, fiscales, funcionarios de enlace y peritos forenses, además del aseguramiento, preservación, documentación y cus- 
todia de todas las evidencias desde la escena del delito hasta la etapa del juicio. Del cumplimiento de esta metodología depende que el examen minucioso de los tejidos blandos y duros que conforman el sistema estomatognático, en busca de evidencia física y/o lesiones que, documentadas, puedan contribuir a establecer la identidad de un individuo, desvirtuar o confirmar un testimonio, o vincular de manera objetiva a un victimario con la víctima y con la escena.

Por esta razón y tal como lo manifiesta la Ley, ${ }^{7,8}$ el odontólogo podrá ser auxiliar de la justicia como funcionario público o como perito designado de acuerdo con los requerimientos de la autoridad competente; por tanto, la odontología como profesión, sus conocimientos y su práctica, son consideradas como una fuente valiosa de datos que en el contexto forense se emplean en procesos de identificación de cadáveres y restos humanos cuyo reconocimiento a partir del análisis del sistema estomatognático, permitirá lograr su identificación positiva y satisfacer una necesidad social básica, facilitar la identificación de un individuo y aportar pruebas periciales preliminares que puedan guiar un procedimiento judicial. No obstante, sufraga otro proceso de igual importancia, el ético y el moral. Permitirle a una familia que cumpla con la costumbre humana del luto y la sepultura del ser querido se convierte en un deber del que la investigación forense no puede ser ajena, de tal forma que contribuya directamente, tanto al procedimiento judicial como al mejoramiento de la calidad de vida de los seres humanos, en donde el culto a los muertos se establece como un derecho digno de ser fundamental, tal como lo refiere el Convenio de Ginebra del 12 de agosto de 1949 en su protocolo adicional: "el derecho que asiste a las familias de conocer la suerte de sus miembros, de recuperar e identificar sus muertos".

No obstante, surge uno de los principales inconvenientes en que se ve envuelta la odontología forense para realizar dicho proceso de identificación al recolectar la información antemortem necesaria para el cotejo como lo es la gran cantidad de personas que no tienen acceso a los servicios de salud oral o no los emplea, por lo que no se cuenta con registros odontológicos antemortem de todos los colombianos. Del mismo modo, se perciben dificultades en la lectura y análisis de las Historias Clínicas debido a la ausencia de información y a una serie de situaciones obvias (como archivo de las Historias Clínicas con los respectivos exámenes radiográficos y órdenes de laboratorio clínico y dental, diligenciamiento del odontograma final, descripción de aparatos pro- tésicos entre otros) para el ejercicio de los técnicos, auxiliares, profesionales y especialistas de la salud oral que hacen que se reduzcan las posibilidades de identificación al momento del cotejo; tal es el caso de la no realización de la Carta Dental, la omisión de la toma de la huella digital, el desecho de las Historias Clínicas antes de tiempo, la pérdida de las radiografías y exámenes complementarios, la ausencia de descripciones de los aspectos estomatológicos individuales y la falta de registro de los tratamientos efectuados, por citar los más comunes ${ }^{16}$.

Ante esta situación, se ha realizado el reporte de este caso y se ha sustentado en la literatura científica la importancia de la odontología forense, con el fin de llamar la atención y demostrar a todo el personal de la salud implicado en la elaboración y diligenciamiento de la Historia Clínica Odontológica, que un proceso de identificación es positivo cuando se colabora oportunamente con la entidad competente y se elaboran de forma correcta y completa, la Historia Clínica y la Carta Dental, de tal manera que el odontólogo forense puede realizar el cotejo ante-postmortem y establecer objetivamente la identidad fehaciente de un cadáver o de unos restos humanos. Por tal razón, es importante indicar que la Resolución 1995 de $1999,{ }^{50}$ ha sido modificada mediante la Resolución 1715 de 2005 en lo que concierne al tiempo de retención y conservación de las Historias Clínicas, ${ }^{51}$ el cual, a partir de la fecha de la última atención, debe ser de mínimo tres años en el archivo de gestión del prestador de servicios de salud y mínimo siete años en el archivo central; luego de transcurrido este tiempo, las historias pueden ser destruidas de acuerdo con las instrucciones dadas en dichas resoluciones.

\section{BIBLIOGRAFÍA}

1. Valdés Y, Casas JA. Odontología legal y forense. En: Herazo B. Odontología Forense. Santafé de Bogotá: Ecoe Editores; 1995. p 85 - 111.

2. Standish SM, Stimson PG. Objetivos de la Odontología Forense. En Standish SM, Stimson PG, directores huéspedes, Clínicas Odontológicas de Norteamérica: odontología forense. Primera edición. Interamericana: México; 1977. p. 3 - 5 .

3. Guerra AS. Odontoestomatología forense. Santafé de Bogotá: Ecoe Editores; 2002.

4. Montiel-Sosa J. Manuales de crimininalística. Noriega editores: México; 1994.

5. Moya V, Roldan B, Sánchez J A. Odontología Legal y Forense. Barcelona: Editorial Masson S. A.; 1994.

6. Moreno S, Moreno F. Antropología dental: una herramienta valiosa para fines forenses. Revista Estomatología 2002; 10: $29-42$. 
7. República de Colombia. Ley 38/1993 de 15 de Enero. Ley por la cual se unifica el sistema de dactiloscopia y se adopta la Carta Dental para fines de identificación. Diario Oficial de la República de Colombia, No. 40724, (19-01-1993).

8. República de Colombia. Ley 35/1989 de 08 de Marzo. Código de Ética del odontólogo colombiano. Tercera edición comentada. Cali: Tribunal de Ética Odontológica Seccional Valle del Cauca; 2001.

9. República de Colombia. Ley $600 / 2000$ de 24 de Julio. Nuevo Código de Procedimiento Penal. Edición actualizada. Santa fe de Bogotá: Momo Editores; 2000.

10. Sweet D. ¿Por qué es necesario un odontólogo para la identificación? En: Fixot RH. Clínicas Odontológicas de Norteamérica: Odontología forense. Interamericana: México; 2001. p. 245-257.

11. Estupiñán DM, Fontanilla GA. Papel del odontólogo en la detección y prevención del maltrato infantil. Univ Odontol 2002; 22: 90 - 97.

12. Marín L, Moreno F. Odontología forense: identificación odontológica, reporte de casos. Revista Estomatología, 2003: $11: 41$ - 49 .

13. Marín L, Moreno F. Odontología forense: identificación odontológica de individuos quemados, reporte de dos casos. Revista Estomatología 2004; 12: 57 - 70.

14. Orjuela CE. Odontología forense. En: Téllez NR. Medicina forense: manual integrado. Santafé de Bogotá: Universidad Nacional de Colombia; 2002. p. 361-365.

15. Orjuela CE, Duque MA, Velosa G, Carreño MI, Constantín AE. Guía Práctica para el Dictamen Odontológico Forense. Santafé de Bogotá: Instituto Nacional de Medicina Legal y Ciencias Forenses; 2004.

16. Avon SL. Forensic odontology: the roles and responsibilities of the dentist. J Can Dent Assoc 2004; 70: 453 - 458.

17. Rodríguez JV. Dientes y diversidad humana: avances de la antropología dental. Primera edición. Santafé de Bogotá: Universidad Nacional de Colombia; 2003.

18. Rodríguez JV. En la antropología forense en la identificación humana. Primera edición. Santafe de Bogotá: Universidad Nacional de Colombia; 2004

19. Sweet D, Hildebrand DP, Phillips D. Identification of a skeleton using DNA from teeth and PAP semear. J Forens Sci 1999; 44:630.

20. Pretty A, Sweet ID. A look at forensic dentistry Part 1: The role of teeth in the determination of human identity. $\mathrm{Br}$ Dent J 2001; 190: 359 - 366.

21. Rodríguez JV. Introducción a la antropología dental. Cuadernos de Antropología 1989; 19: 1-41.

22. Scott GC, Turner II CG. The anthropology of modern human teeth: dental morphology and its variation in recent human populations. London: Cambridge University Press; 1997.

23. Zoubov AA. La antropología dental y la práctica forense. Maguaré 1998; 13: 243-252.

24. Moreno F, Moreno SM, Díaz CA, Bustos EA, Rodríguez JV. Prevalencia y variabilidad de ocho rasgos morfológicos dentales en jóvenes de tres colegios de Cali, 2002. Colomb Med 2004; 35 (Supl 1): 16 -23.

25. Edgar HJ. Prediction of race using characteristics of dental morphology. J Forensic Sci 2005; 50: 1 - 5.

26. Moreno SM, Moreno F. Eight non-metric dental traits in alive racially mixed population from Cali, Colombia. Inter J Dent Anthropol 2005; 6: 14 - 25.
27. Aguirre L, Castillo D, Solarte D, Moreno F. Frequency and variability of five non-metric dental crown traits in the primary and permanent dentitions of a racially mixed population from Cali, Colombia. Dent Anthropol 2006; 19: $39-47$.

28. Mertz CA. Identificación dental. En: Standish SM, Stimson PG. Clínicas Odontológicas de Norteamérica. Odontología Forense. Editorial Interamericana: México; 1997. p. 47 - 67.

29. Cárdenas F. Dieta prehispánica en poblaciones arqueológicas Muiscas. En: Enciso B, Therrien M, Bioantropología de la Sabana de Bogotá. Santa Fe de Bogotá: Colcultura, 1996. p. 88 - 109.

30. Rodríguez JV. Los Chibchas: pobladores antiguos de los Andes Orientales. Aspectos bioantropológicos.Santafé de Bogotá: Universidad Nacional de Colombia; 1999.

31. Formicola V. Interproximal grooving of teeth: additional evidence and interpretation. Current Anthropology 1988; 29: 663 - 671

32. Hillson S. Dental Anthropology. London: Cambridge University Press; 1996.

33. Beltrán R. Las mutilaciones dentarias en Mesoamérica. Revista de la Asociación Dental Mexicana 2002; 59: 28 $-33$.

34. De Urbiola AI, Viñals IH. Algunas consideraciones acerca de los piercings orales. Avances en Odontoestomatología 2005; 21: 259 - 269.

35. Krishan K, Vashisht, RN, Vij K. Teeth in personal identification. Medico-Legal Update 1997; 2: 9 - 11.

36. Whittaker DK. Forensic dentistry in the identification of victims and assailants. J Clin Forensic Med 1995; 2: 145-151.

37. Guerra AS, Pérez GE. Importancia de la radiografía oral en el diagnóstico de la edad en restos óseos humanos. Revista de la Federación Odontológica Colombiana 2001; 200: 51 - 59.

38. Soomer h, Lincoln MJ, Ranta R, Penttilä A, Leibur E. Dentists' qualifications affect the accuracy of radiographic identification. J Forensic Sci 2003; 48: 1 - 6.

39. Carmichael WW. Significance of the recent extraction to the postmortem dental identification: A case Study. J Forensic Sci 2002; 47: 1-3.

40. Adams BJ. Establishing personal identification based on specific patterns of missing, filled, and unrestored teeth. J Forensic Sci 2003; 48: 487 - 496.

41. Gil P, Miquel M, Negre M, Polo M, Villalaín J. Pseudopatología tafonómica en restos óseos arqueológicos [en línea]. Departamento de Medicina y Medicina Legal de la Universidad de Valencia: UpToDate; 1996 [fecha de acceso: noviembre de 2006]. URL disponible en http://147.96.1.15/ info/aep/boletin/actas/38.pdf

42. Hochrein MJ. Buried crime scene evidence: the application of forensic geotaphonomy in forensic archaeology. En: Stimson PG, Mertz CA. Forensic Dentistry. London: CRC Press; 1997.

43. Merlati G, Savio C, Danesino P, Fassina G, Menghini P. Further study of restored and unrestored teeth subjected to high temperatures. J Forensic Odontostomatol 2004; 22: $17-24$.

44. Ferreira JL, Espina AL, Barrios FA, Mavaréz MG. Conservación de las estructuras orales y faciales del cadáver quemado. Ciencia Odontológica 2005; 2: 58 - 65. 
45. Mazza A, Merlati G, Savio C, Fassina G, Menghini P, Danesino P. Observations on dental structures when placed in contact with acids: experimental studies to aid identification processes. J Forensic Sci 2005; 50: 406 - 410.

46. Toribio T, Rivero M. Método para la estimación del número de enterramiento en un sitio funerario. Rev. Cub. Estomatol [revista en línea] 1996. [accedido en septiembre de 2005]; 33 (2). URL disponible en: http://bvs.sld.cu/revistas/est/vol33_2_96/est01296.htm

47. Rodríguez JV. Estimación de la edad, sexo, ancestros y estatura en restos óseos humanos. En: Herazo B, editor. Odontología Forense. Ecoe Editores: Santafe de Bogotá; 1995. p. 1 - 50.

48. Archer MS, Bassed RB, Briggs CA, Lynch MJ. Social isolation and delayed discovery of bodies in houses: The value of forensic pathology, anthropology, odontology and entomology in the medico-legal investigation Forensic Science International 2005; 151: 259 - 265.

49. American Board of Forensic Odontology. Guidelines for bite mark analysis. J Am Dent Assoc 1986; 112: 383 386.

50. República de Colombia. Resolución 1995/1999 de 08 de Julio. Por la cual se establecen Normas para el Manejo de la Historia Clínica. Diario Oficial de la República de Colombia, No. 43655, (05-08-1999).

51. República de Colombia. Resolución 001715/2005 de 13 de Junio. Por la cual se modifica la Resolución 1995/1999 de 08 de Julio. Diario Oficial de la República de Colombia, No. 45940, (15-06-2005).
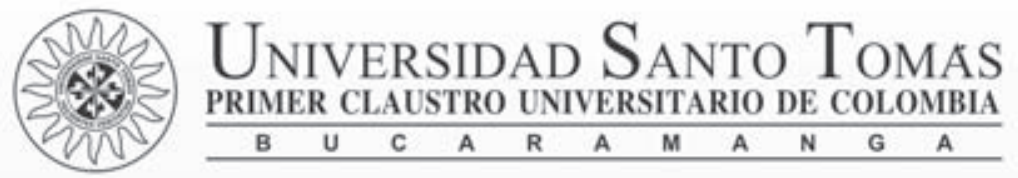

\section{Especializaciones en}

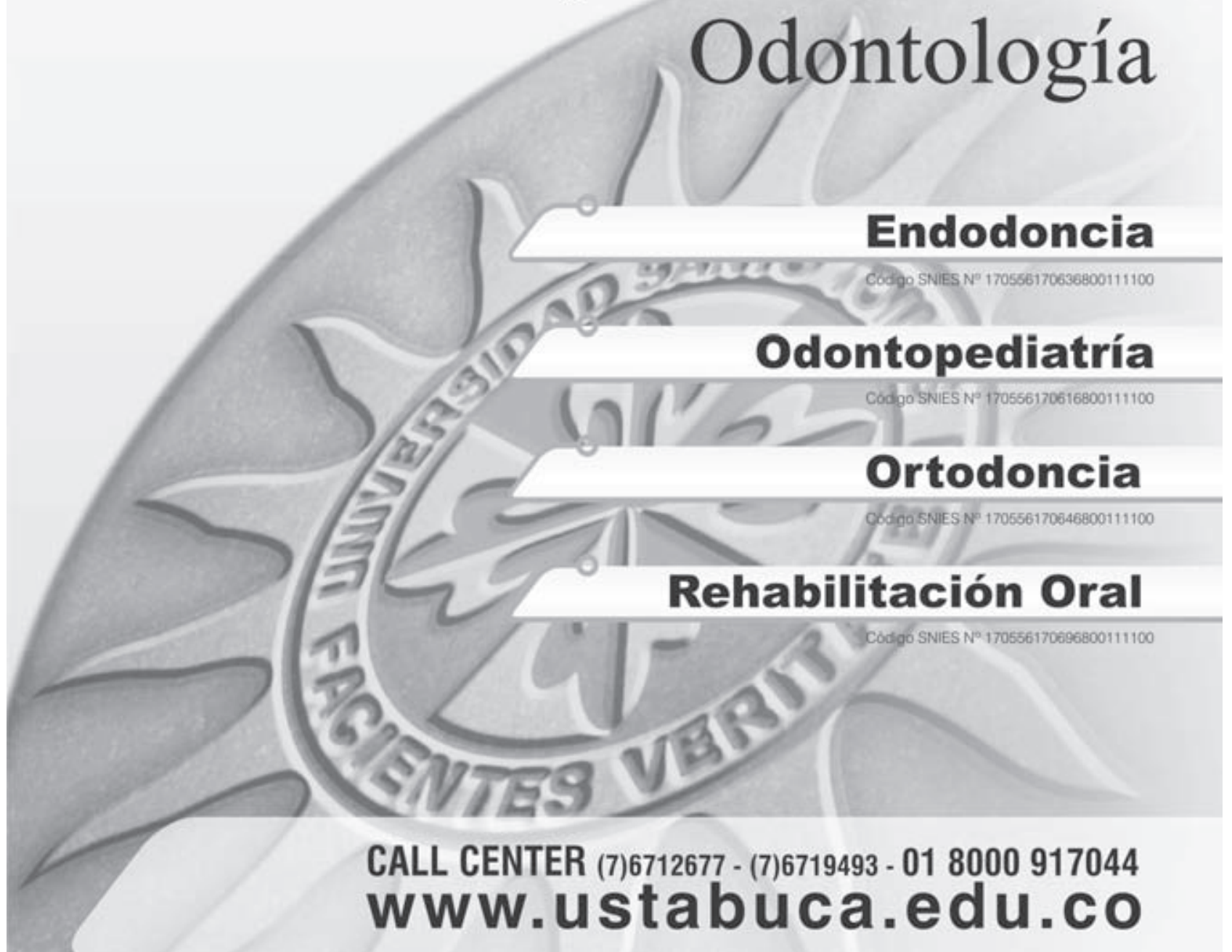

